\title{
Clinicaltrials.gov NCT Number for the Expanded Access Record
}

National Cancer Institute

\section{Source}

National Cancer Institute. Clinicaltrials.gov NCT Number for the Expanded Access

Record. NCI Thesaurus. Code C127792.

The unique alphanumeric identifier for a study with associated expanded access record, as assigned by the clinicaltrials.gov PRS system. 